\title{
Annus physicalis 1932
}

\section{A bumper crop of physical discoveries - something in the (heavy) water?}

A physicist reviewing our century will find a discovery or new insight in every year, but 1932 stands out as a true annus mirabilis. John Dryden, who coined the term to describe 1666, discussed three events: the plague, the fire of London, and the war with the Dutch. The physicists' harvest for 1932 yielded four major discoveries: the neutron, heavy hydrogen, nuclear reactions induced by accelerated particles, and the positive electron, or positron.

Was there anything mysterious and special about 1932? I do not think so, but during the 1920s study of the behaviour of electrons in atoms led to the creation of quantum mechanics. The time was ripe for physicists to go further - it is not surprising that a number of fundamental discoveries were

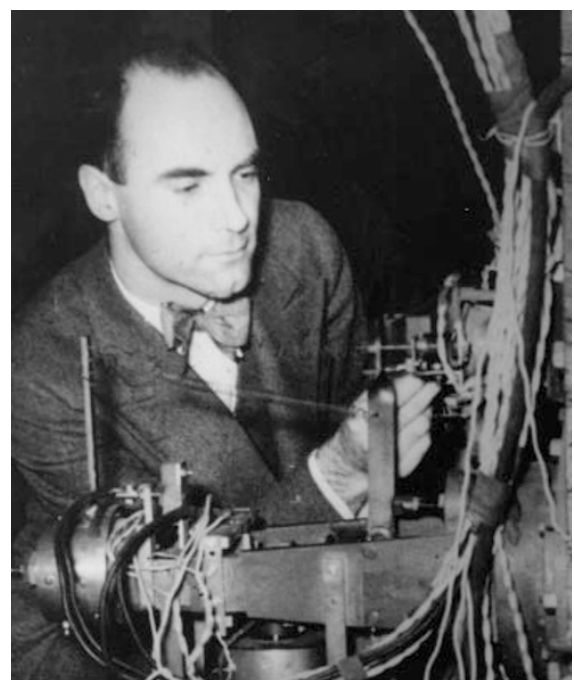

\section{H. B. G. Casimir}

made in the 1930s, but for four of them to come in one year is just a matter of chance.

The existence of the neutron, a particle without electric charge and with roughly the same mass as the nucleus of the hydrogen atom (the proton), had been postulated by Ernest Rutherford. It was discovered at Cambridge in 1932 by James Chadwick, who created beams of neutrons by irradiating beryllium with alpha particles. It was at once clear that the existence of the neutron led to a simple model for nuclear structure. Nuclei were henceforth regarded as compounds of protons and neutrons. The simplest case is one proton and one neutron. This particle was called deuteron, $D$. Its oxide, $\mathrm{D}_{2} \mathrm{O}$, is the molecule of heavy water. It was found in 1932 that about one part in six thousand of normal water is heavy water.

Because neutrons have no charge, they are not repelled by the electric fields of other nuclei. Therefore they can enter them and produce all manner of nuclear reactions. By giving a proton sufficient energy — in effect, using brute force - it is also possible for it to overcome electrical repulsion. This was done by John Cockcroft and Ernest Walton, also at

Positive images: the first positron track (top), discovered by Anderson (left), confirmed Dirac's predictions (below).

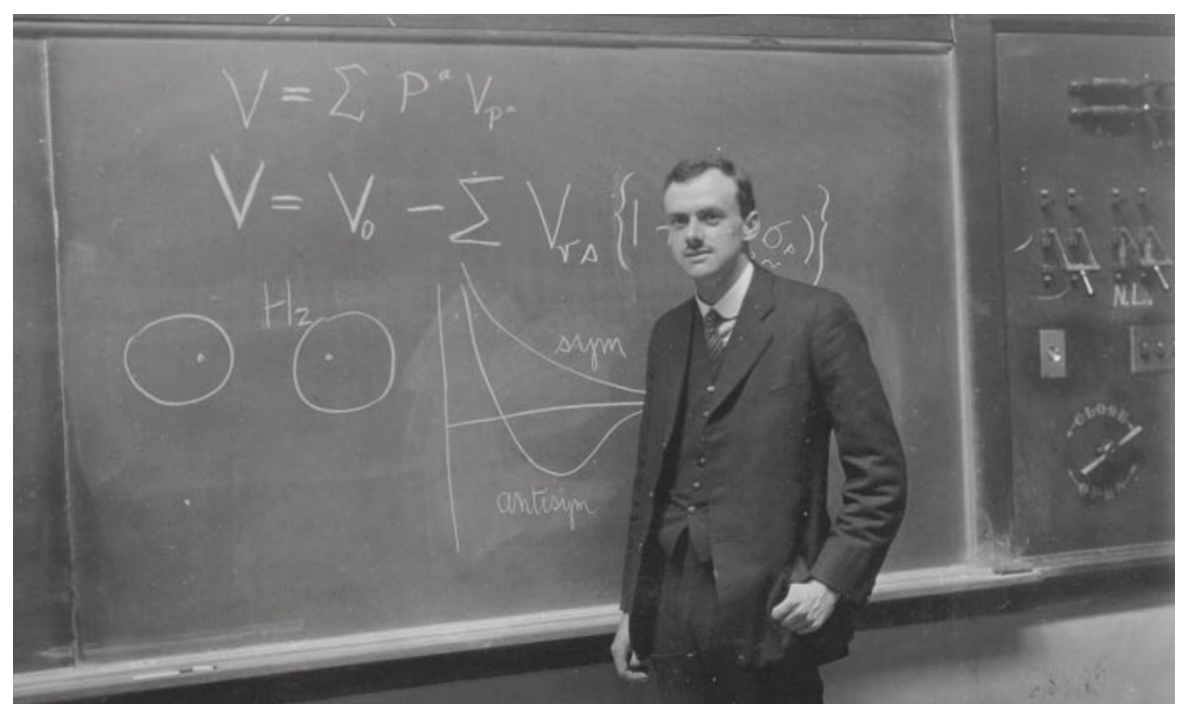

Cambridge. With a 700-kilovolt high-voltage generator they accelerated protons and let them fall on lithium. They showed that some of the lithium nuclei broke in two - and proudly announced that they had split the atom. So began the era of big machines. The high-voltage generators were succeeded by cyclotrons, the cyclotrons by even more powerful apparatus. Neutron, deuteron and accelerators formed the beginning of an extremely active period of nuclear physics that led to nuclear energy and weapons.

The fourth discovery, the positron, was made by Carl Anderson, using what could be called the astronomical method - examining phenomena outside our control, in this case the so-called cosmic radiation.

Here we see clearly the difference between physics and astronomy. The physicist can arrange and manipulate the things he or she studies, the astronomer cannot. Astronomers can observe in many ways, using all the resources of physics and technology, and may even build an observatory on the Moon, but they cannot influence the structure or behaviour of planets, let alone stars. Nor can they influence the cosmic radiation.

Apart from the sign of its charge, a positron is identical to a normal electron. Entirely new, however, is that a positronelectron pair can be created by collision processes in empty space, and that the pair can disappear. The partners annihilate one another, leaving their energy behind as electromagnetic radiation. Again, the positron's existence and behaviour were predicted, this time by Paul Dirac.

The positron was the first short-lived particle to be created out of empty space. It is a prelude to the high-energy physics that blossomed after the Second World War. Today, ever more complicated machines grant us fleeting glimpses of whole families of shortlived particles, many of them predicted by theory. Sometimes it almost appears that the theories are not a description of a nearly inaccessible reality, but that so-called reality is a result of the theory.

In any case, the limits of this strange new world are not yet in sight.

H. B. G. Casimir is at De Zegge 7, 5591 TT Heeze, The Netherlands. 